\title{
GIDE is a mitochondrial E3 ubiquitin ligase that induces apoptosis and slows growth
}

Bicheng Zhang ${ }^{1, *}$, Jun Huang ${ }^{2,}$, Hong-Liang $\mathrm{Li}^{3,}{ }^{*}$, Ting Liu ${ }^{2}$, Yan-Yi Wang ${ }^{4}$, Paul Waterman ${ }^{1}$, Ai-Ping Mao ${ }^{4}$, Liang-Guo $\mathrm{Xu}^{1}$, Zhonghe Zhai ${ }^{2}$, Depei Liu ${ }^{3, \S}$, Philippa Marrack ${ }^{1, \S}$, Hong-Bing Shu, ${ }^{4, \S}$

${ }^{1}$ HHMI, National Jewish Medical and Research Center, Denver, CO 80206, USA $;{ }^{2}$ College of Life Sciences, Peking University, Beijing 100871, China; ${ }^{3}$ Institute of Basic Medical Sciences, Chinese Academy of Medical Sciences, Beijing 100005, China; ${ }^{4}$ College of Life Sciences, Wuhan University, Wuhan 430072, China

Here, we report the identification of GIDE, a mitochondrially located E3 ubiquitin ligase. GIDE contains a C-terminal RING finger domain, which is mostly conserved with those of the IAP family members and is required for GIDE's E3 ligase activity. Overexpression of GIDE induces apoptosis via a pathway involving activation of caspases, since caspase inhibitors, XIAP and an inactive mutant of caspase-9 block GIDE-induced apoptosis. GIDE also activates JNK, and blockage of JNK activation inhibits GIDE-induced release of cytochrome c and Smac as well as apoptosis, suggesting that JNK activation precedes release of cytochrome c and Smac and is required for GIDEinduced apoptosis. These pro-apoptotic properties of GIDE require its E3 ligase activity. When somewhat over- or underexpressed, GIDE slows or accelerates cell growth, respectively. These pro-apoptotic or growth inhibition effects of GIDE may account for its absence in tumor cells.

Keywords: GIDE, apoptosis, E3 ligase, mitochondria, caspase, JNK

Cell Research (2008) 18:900-910. doi: 10.1038/cr.2008.75; published online 1 July 2008

\section{Introduction}

Apoptosis is required for normal development in all metazoans. Abnormal regulation of apoptosis leads to various diseases, such as cancer and autoimmune and neurodegenerative diseases. Apoptosis is mediated by activation of caspases, which cleave various cellular substrates and lead to characteristic apoptotic morphological changes [1-3]. Caspases are synthesized as cytoplasmic zymogen precursors and are activated in a hierarchical pattern in which downstream caspases are activated by upstream caspases [1-3]. The upstream caspases are activated through distinct mechanisms. For example, it has been proposed that the death receptor associated caspase- 8 is activated by so-called "induced proximity" [4].

\footnotetext{
*These three authors contributed equally to this work. $\S$ These three authors are co-corresponding authors. Correspondence: Hong-Bing Shu

Tel/Fax: +86-27-68753780

E-mail: shuh@whu.edu.cn

Received 22 April 2008; revised 24 April 2008; accepted 24 April 2008; published online 1 July 2008
}

In most cases, however, activation of the initial caspases involves mitochondria. Upon stimulation by various death signals, the outer membrane of the mitochondrion is permeabilized, resulting in the release of molecules, including cytochrome $c$, Smac/DIABLO, Omi/HtrA and GSPT1/eRF3, to the cytosol. Released cytochrome $c$ causes cell death by binding to Apaf-1, an event that leads to recruitment and activation of pro-caspase-9 [5]. Unlike cytochrome $c$, mitochondrial-released Smac/DIABLO, Omi/HtrA2 and GSPT1/eRF3 induce caspase activation and apoptosis through their interactions with proteins of the IAP family [6-8].

IAP proteins are universally present in organisms from yeast to humans. All IAPs contain at least one baculovirus IAP repeat (BIR) domain and most also contain a C-terminal RING finger domain, which has E3 ubiquitin ligase activity. Although some IAP proteins have other activities, most IAPs inhibit apoptosis [9]. Among the human IAPs, XIAP is the most extensively studied and the most potent inhibitor of apoptosis. XIAP binds to active caspase- 9 with high affinity, and with lower affinity to caspase-3 and -7. Binding of XIAP to active caspases prevents their cleavage of cellular substrates, and thus 
inhibits apoptosis [10]. XIAP may also target active caspase-3 for ubiquitination and degradation by the proteasome, thereby inhibiting apoptosis [11]. Binding of mitochondrial-released Smac/Diablo, Omi/HtrA2 and GSPT1/eRF3 to IAPs causes release of active caspases from inhibitory IAPs and/or inhibition of ubiquitination and degradation of caspases, and therefore promotes apoptosis [9]. Recently, it has been shown that XIAP and the baculovirus Op-IAP can ubiquitinate Smac/Diablo and target it for degradation by the proteasome, suggesting that IAPs can also inhibit apoptosis through degradation of their antagonists, rather than by directly inhibiting caspases [12]. Whatever the mechanisms, the balance between mitochondrial-released death factors and cytosolic IAPs appears to be critically involved in the modulation of apoptosis.

Many extrinsic and intrinsic signals can trigger apoptosis. For example, JNK, a mitogen-activated protein ki- nase (MAPK) that is activated by stimuli that cause cell stress, such as DNA damaging reagents, UV light and proinflammatory cytokines, is pro-apoptotic under some circumstances in some cell types [13, 14]. In Drosophila, the JNK ortholog, DJNK, is required for apoptosis during embryonic patterning of the wing, eye and gut [15]. DJNK phosphorylates DJun, which promotes transcription of Hid and Rpr, two proteins that bind to the Drosophila IAP ortholog DIAP1 and prevent DIAP1 from inhibiting the Drosophila caspase, DRONC, thus triggering apoptosis [16].

In mammals, there is evidence for both pro-apoptotic and anti-apoptotic roles of JNK. JNK activity is required for apoptosis of PC12 neuronal cells induced by NGF withdrawal, of MEFs in response to many stimuli and of thymocytes in response to T cell receptor ligation [17-19]. However, knockout of JNK1 and JNK2 in mice can increase apoptosis in the brain during embryonic develop-

A

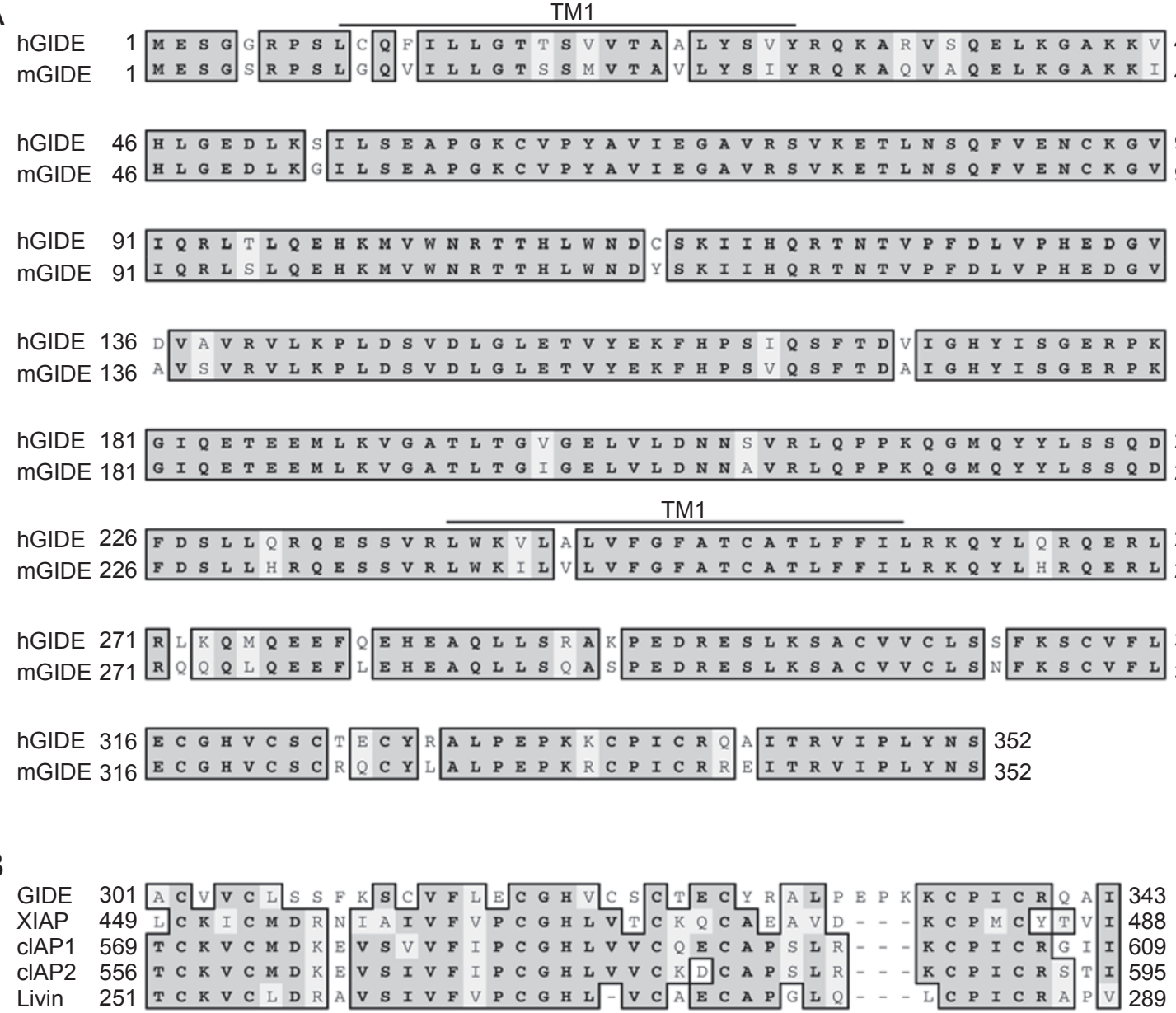

Figure 1 Sequence and structural analysis of GIDE. (A) Alignment of human and mouse GIDE amino acid sequences. The putative transmembrane regions (TM) are indicated. (B) Alignment of conserved RING-fingers of human GIDE, XIAP, c-IAP1, C-IAP2 and Livin. 
ment [20].

In this study, we identified a mitochondrial protein, GIDE, that contains a RING finger domain homologous to those of the IAP family members. GIDE slows the growth of cells and, when overexpressed, induces apoptosis. GIDE has E3 ubiquitin ligase activity, which is required for its ability to induce apoptosis.

\section{Results}

A

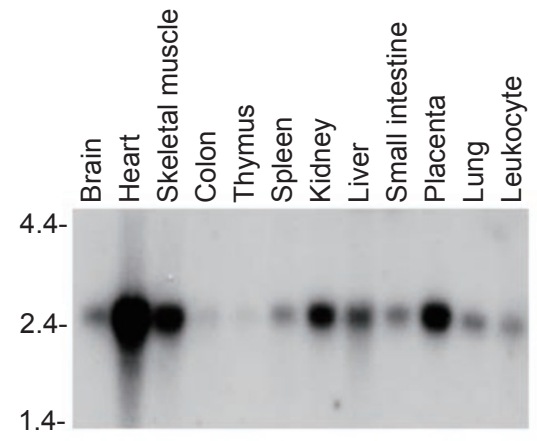

Identification and tissue distribution of GIDE

Due to our interest in the anti-apoptotic functions of the RING finger domains of IAPs, we searched the EST databases for novel proteins that contained C-terminal RING finger domains like those of the IAPs. We thus identified the novel protein GIDE (for Growth Inhibition and Death E3 Ligase); through alignment of the available cDNA and EST sequences, we obtained an $\sim 2.5 \mathrm{~kb}$ human GIDE cDNA sequence that encodes a 352 aa protein. $\mathrm{Hu}-$ man GIDE displays an approximately $90 \%$ amino acid

B
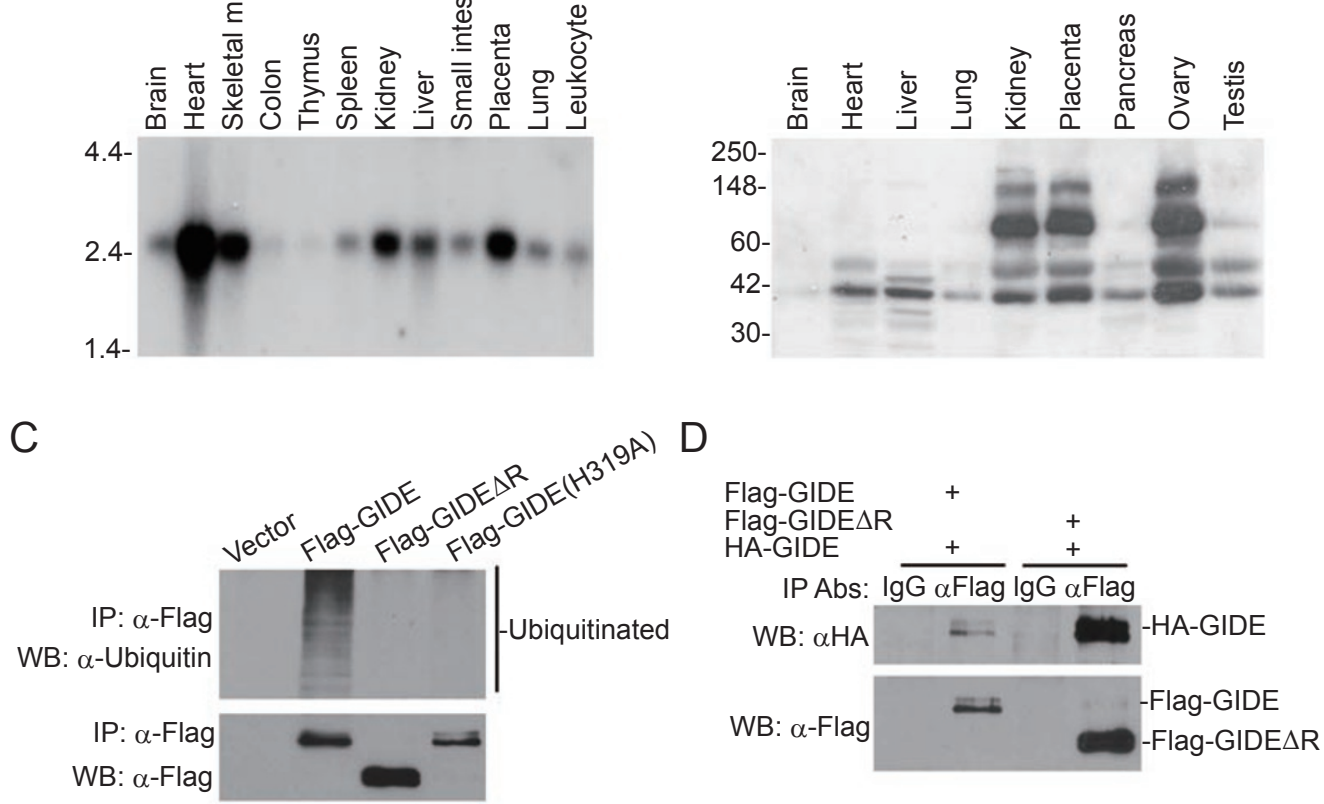

E

$\mathrm{F}$
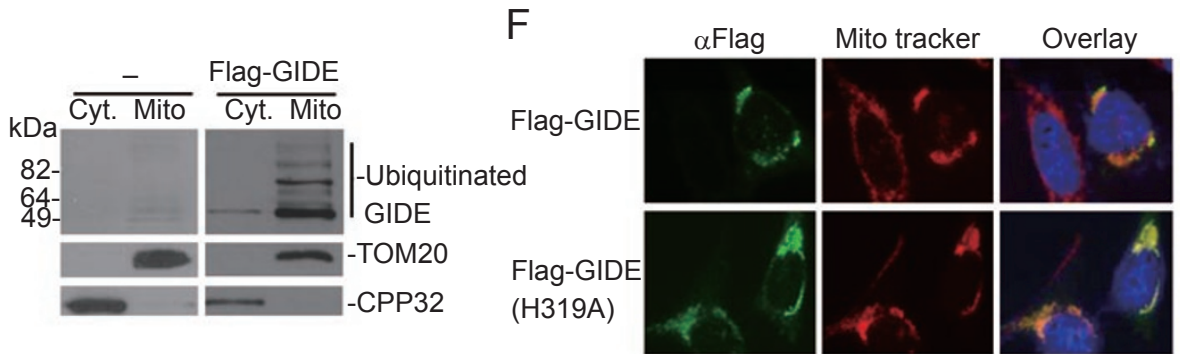

Figure 2 Expression, ubiquitination and localization of GIDE. (A) Northern blot analysis of human GIDE mRNA expression in various tissues. (B) Western blot analysis of human GIDE protein expression in various tissues. (C) GIDE is auto-ubiquitinated. 293 cells were transfected with the indicated Flag-tagged plasmids. Cell lysates were immunoprecipitated with anti-Flag antibody, the immunoprecipitates were analyzed by western blot with anti-ubiquitin (upper panel) and anti-Flag (lower panel) antibodies. (D) GIDE forms oligomers. 293 cells were transfected with the indicated GIDE or its mutant plasmids. Cell lysates were immunoprecipitated with anti-Flag antibody or control mouse IgG; the immunoprecipitates were analyzed by western blot with anti-HA antibody. (E) Biochemical study of GIDE localization. 293 cells were transfected with Flag-GIDE or left untransfected for $12 \mathrm{~h}$. Mitochondrial and cytosolic fractions were isolated and analyzed by western blots with the indicated antibodies. (F) Immunofluorescent staining of GIDE and its mutant GIDE(H319A). HeLa cells were transfected with the indicated plasmids and stained with the indicated antibodies. 
A
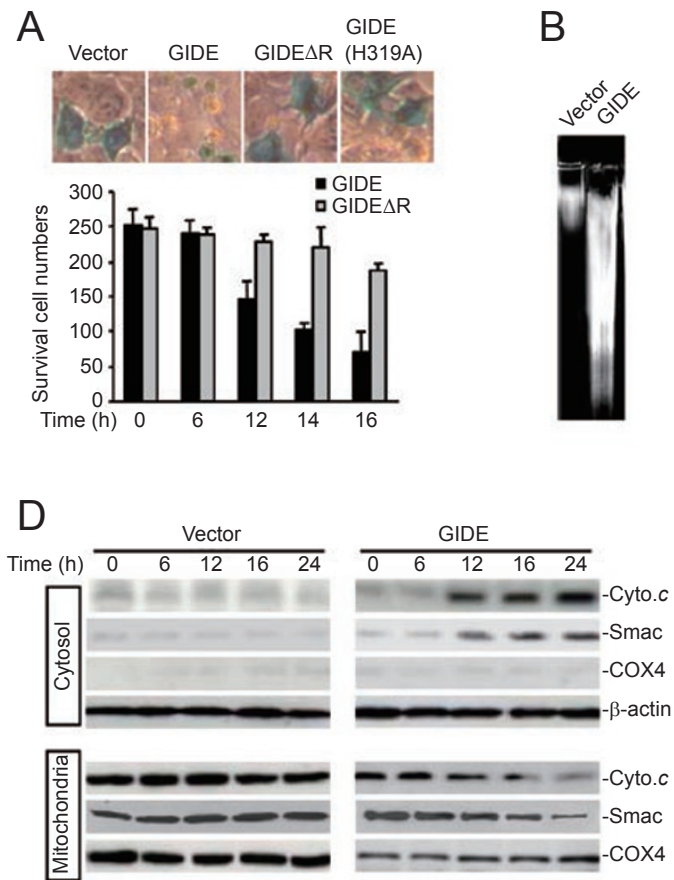

B

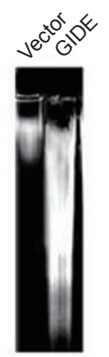

$\mathrm{E}$

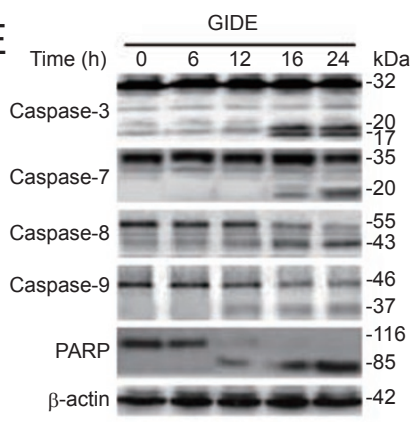

Figure 3 GIDE induces apoptosis in 293 cells. (A) The effects of GIDE and its mutants on cell survival. 293 cells $\left(\sim 1 \times 10^{5}\right)$ were transfected with $0.1 \mu \mathrm{g}$ of CMV- $\beta$-gal plasmid and $0.5 \mu \mathrm{g}$ of the indicated expression plasmids. At $16 \mathrm{~h}$ after transfection, cells were stained with X-gal and photographed (upper panels), and surviving blue cells were counted (lower panel). (B) Overexpression of GIDE causes DNA fragmentation. 293 cells $\left(\sim 1 \times 10^{6}\right)$ were transfected with $10 \mu \mathrm{g}$ of the indicated plasmids. At $16 \mathrm{~h}$ after transfection, DNA was isolated from the transfected cells and analyzed by $2 \%$ agarose gel. (C) Overexpression causes loss of mitochondrial membrane potential. 293 cells $\left(\sim 2 \times 10^{5}\right)$ were transfected with $1 \mu \mathrm{g}$ of the indicated expression plasmids. At $16 \mathrm{~h}$ after transfection, cells were stained with Rh123 $(10 \mu \mathrm{g} / \mathrm{ml})$ at $37^{\circ} \mathrm{C}$ for 30 min and then washed three times with PBS (+). The mitochondrial membrane potential was analyzed by flow cytometry. (D) Overexpression of GIDE causes release of cytochrome $c$ and Smac from mitochondria to the cytosol. 293 cells were transfected with GIDE or control plasmid for the indicated times. The cell lysates were fractionated and analyzed by western blots with the indicated antibodies. (E) Overexpression of GIDE causes activation of caspases. 293 cells were transfected with GIDE for the indicated times. Cell lysates were analyzed by western blots with the indicated antibodies.

identity with its rat and mouse orthologs. The C-terminus of human GIDE contains a RING finger domain at aa 301-343 that resembles those of IAPs (Figure 1 and data not shown). Except for the RING finger domain, GIDE is not homologous to any known proteins.

To determine the expression pattern of GIDE, we performed northern blot analysis with a multiple human tissue mRNA blot. The data suggest that human GIDE mRNA is expressed, as an $\sim 2.5 \mathrm{~kb}$ transcript, in most tissues. Expression of GIDE mRNA is relatively abundant in the heart, skeletal muscle, placenta, kidney and liver, but barely detectable in the colon and thymus (Figure 2A).

We raised a rabbit polyclonal antibody against human GIDE and determined the expression of GIDE protein in human tissues. A band of $\sim 41 \mathrm{kDa}$, the predicted molecular weight of GIDE, was detected in all tissues (Figure 2B). Interestingly, several other higher molecular weight bands were detected in certain tissues, including kidney, placenta, ovary and testis (Figure 2B). These bands are probably ubiquitinated GIDE, based on our observations that GIDE has E3 ubiquitin ligase activity and is extensively auto-ubiquitinated when overexpressed in mammalian cells (Figure 2C). These bands are not derived from alternatively spliced GIDE mRNA, because only one band was detected in northern blot analysis (Figure 2A) and the size of the transcript matches the size of the cDNA we obtained. The bands do not represent proteins that are non-specifically recognized by the GIDE antibody, as this antibody did not detect any proteins in multiple cancer cell lines (see Discussion).

\section{GIDE has E3 ubiquitin ligase activity and is localized to} the mitochondria

Since the RING finger domains of the IAP family members confer E3 ubiquitin ligase activities, we deter- 
mined whether GIDE also has this activity. When overexpressed in 293 cells, GIDE was strongly ubiquitinated (Figure 2C). In these experiments, GIDE $\Delta \mathrm{R}$, a mutant of GIDE in which the C-terminal RING finger is deleted, as well as GIDE(H319A), a mutant in which the crucial histidine residue in the RING finger is mutated to alanine, were not ubiquitinated (Figure 2C).

Consistent with its ability to self-ubiquitinate, GIDE formed homo-oligomers (Figure 2D). A GIDE mutant lacking its RING finger could still interact with wildtype GIDE (Figure 2D), suggesting that the N-terminal domain of GIDE mediates its oligomerization. Taken together, these data indicate that the RING finger domain and the N-terminal domain of GIDE are required for its E3 ligase activity and oligomerization, respectively.

Structural analysis indicated that GIDE contains two transmembrane regions (Figure 1), suggesting that GIDE is probably localized to membrane organelles. To test this, we performed cellular fractionation and immuno- fluorescent staining experiments. GIDE was primarily localized in mitochondria (Figure $2 \mathrm{E}$ and $2 \mathrm{~F}$ ). The ligase inactive mutant of GIDE (H319A) was also localized in mitochondria (Figure $2 \mathrm{~F}$ ), suggesting that ubiquitination of GIDE does not affect its localization.

GIDE induces apoptosis through the intrinsic mitochondrial-dependent apoptotic pathways

Since GIDE contains a C-terminal RING finger domain that is conserved with those of IAP family proteins, we determined whether GIDE plays a role in apoptosis. Surprisingly, overexpression of GIDE caused massive cellular condensation, round-up, detachment from the dish and DNA fragmentation, phenotypes typical of apoptotic cells (Figure 3A). These phenotypes were observed $12 \mathrm{~h}$ after transfection (Figure $3 \mathrm{~A}$ ) and were dosedependent (data not shown). Overexpression of GIDE $\Delta \mathrm{R}$ or GIDE(H319A) had no significant apoptotic effects (Figure 3A). These data indicate that GIDE can induce
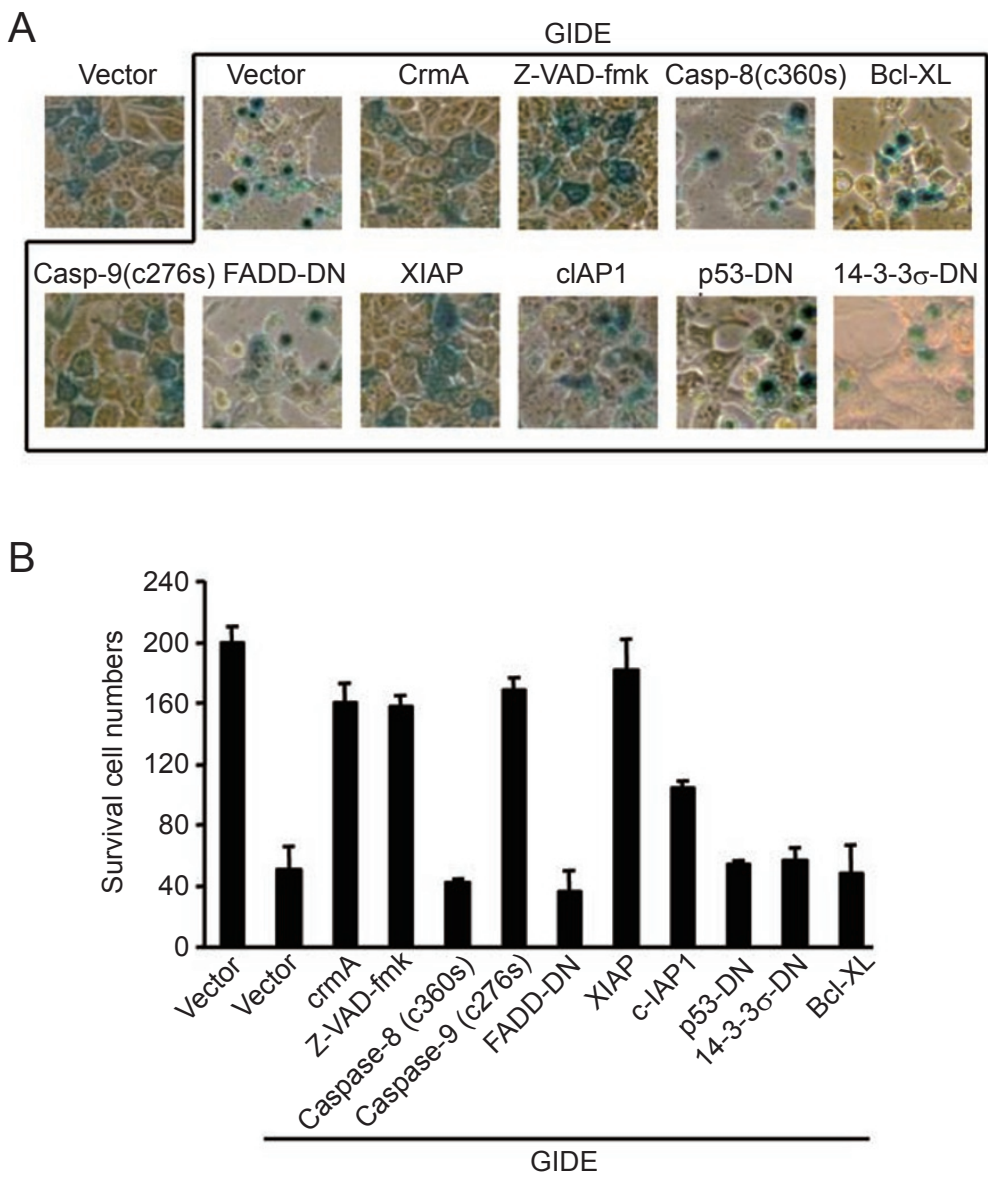

Figure 4 Effects of various apoptosis inhibitors on GIDE-induced apoptosis. 293 cells were transfected with GIDE and the indicated plasmids, or treated with the caspase inhibitor Z-VAD-fmk. At $16 \mathrm{~h}$ after transfection, cells were stained with X-gal and photographed $(A)$, and surviving blue cells were counted $(B)$. 
rapid apoptosis and that the E3 ligase activity of GIDE is important for this activity.

To determine the mechanisms responsible for GIDEinduced apoptosis, we examined the effects of GIDE on the mitochondrial membrane potential, cytochrome $c$ and Smac release, and caspase activation. Overexpression of GIDE caused dramatic loss of mitochondrial membrane potential (Figure 3C) and release of mitochondrial cytochrome $c$ and Smac into the cytosol (Figure 3D). In contrast, the mitochondrial protein $\mathrm{COX} 4$, which is not released from damaged mitochondria and was used as a control, was not released into the cytosol (Figure 3D). Consistent with these observations, overexpression of GIDE caused processing of pro-caspase- $3,-7,-8$, and -9 , as well as the classic caspase substrate PARP (Figure $3 \mathrm{E})$. In these experiments, it seems that caspase- 9 and -8 were processed earlier than caspase- 3 and -7 (12 vs. $16 \mathrm{~h}$ after transfection), suggesting that caspase- 3 and -7 function downstream of caspase- 9 and/or -8 . These data indicate that GIDE activates the intrinsic apoptotic pathways.

\section{GIDE-induced apoptosis is blocked by caspase inhibitors and IAPS}

Various apoptosis inhibitors were used to determine whether cytochrome $c$ and Smac release and caspase activation are required for GIDE-induced apoptosis. In transient transfection experiments, the caspase inhibitory protein crmA and small molecular pan-caspase inhibitor z-VAD-fmk potently inhibited GIDE-induced apoptosis and DNA fragmentation (Figure 4). Interestingly, a caspase-inactive caspase- 9 mutant had the same effects. In these experiments, a caspase-inactive caspase- 8 mutant and FADD dominant negative mutant inhibited TNF-R1(data not shown), but not GIDE-induced apoptosis (Figure 4). Since overexpression of GIDE activated both caspase- 9 and -8 , it is likely that caspase- 9 functions upstream of caspase- 8 and other caspases, and caspase-9, but not one of its downstream caspases, is required for GIDE-induced apoptosis. Alternatively, caspase-8 activation may be a secondary event in GIDE-mediated apoptosis. In these experiments, XIAP also completely inhibited GIDE-induced apoptosis, while cIAP1 had a weaker inhibitory role (Figure 4). These results suggest that GIDE induces apoptosis through caspase-9 activation and that this process is inhibited by IAPs.

A dominant negative mutant of $\mathrm{p} 53$ and Bcl-XL did not inhibit GIDE-induced apoptosis (Figure 4), but inhibited p53RFP- and Bax-mediated apoptosis (data not shown). Consistent with these results, 14-3-3 $\sigma$, a cytoplasmic sequester of Bax, did not block GIDE-induced apoptosis (Figure 4). Moreover, GIDE could induce apoptosis in $\mathrm{Bax}^{-/-} \mathrm{Bak}^{-/}$-deficient mouse embryonic fibroblasts (data not shown). Taken together, these data suggest that GIDE-induced apoptosis is not mediated by the classical Bax/Bak-dependent pathways.

Recently, it has been shown that the E3 ubiquitin ligase Itch promotes degradation of c-FLIP(L) and apoptosis, upon phosphorylation by JNK. In our experiments, we found that GIDE could not ubiquitinate c-FLIP or cause its degradation. In addition, neither c-FLIP(L) nor its short alternative splice form could inhibit GIDEinduced apoptosis (data not shown). These data indicate that GIDE-induced apoptosis is not mediated by degradation of c-FLIP.

GIDE activates JNK through TAK1-dependent pathways

In the course of our study, another group identified GIDE (referred as clone $266 \mathrm{~N}$ ) in a large-scale screen for $\mathrm{NF}-\kappa \mathrm{B}$ activating proteins, but did not report a detailed functional characterization of the protein [21]. We therefore determined whether GIDE can indeed activate $\mathrm{NF}-\kappa \mathrm{B}$. Both GIDE and GIDE $\Delta \mathrm{R}$ weakly activated NF$\kappa \mathrm{B}$ in a dose-dependent manner (Figure 5A). Since IAPs can activate both $\mathrm{NF}-\kappa \mathrm{B}$ and $\mathrm{JNK}[22,23]$, and GIDE is related to IAPs, we determined whether GIDE can also activate JNK. Overexpression of GIDE activated JNK in a dose-dependent manner (Figure 5B). Overexpression of GIDE also activated JNK in kinase assays using GSTc-Jun as a substrate (Figure 5C). Interestingly, deletion of the RING finger domain of GIDE abolished its ability to activate JNK (Figure 5B and 5C), suggesting that GIDE's E3 ligase activity is required for GIDE-induced JNK, but not NF- $\kappa \mathrm{B}$ activation. Previously, it has been shown that many RING finger domain-containing proteins, including TRAF2, TRAF6 and XIAP, activate JNK through MAPKKK (MEKK) family members [23, 24]. We examined whether kinase-inactive mutants of MEKK1, MEKK3, ASK1, TAK1 and TBK1 could inhibit GIDE-induced JNK activation. In these experiments, only the kinase inactive mutant of TAK1, TAK1(K/W), inhibited GIDEinduced JNK activation (Figure 5D). Interestingly, it has been shown that XIAP activates JNK through TAK1 [23]. In reporter assays, GIDE did not activate p38 and ERK, as indicated by CHOP and Elk1 reporter gene assays, respectively (Figure 5E).

Since GIDE activates JNK through TAK1, we examined whether GIDE could interact with TAK1 and found, in transient transfection and co-immunoprecipitation experiments, that it could. Deletion of GIDE's C-terminal RING finger domain did not affect its ability to interact with TAK1 (Figure 5F).

\section{$J N K$ activation is required for GIDE-induced apoptosis}

Since GIDE can activate both NF- $\mathrm{BB}$ and JNK, we 
A

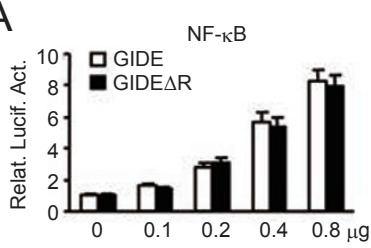

C

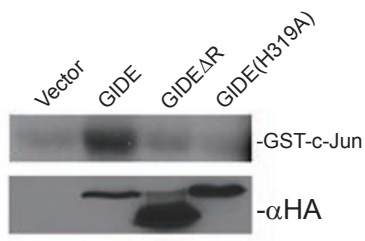

E

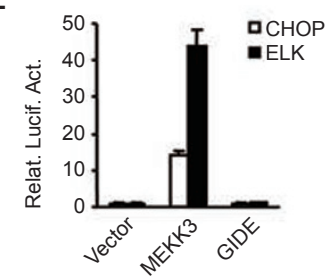

G

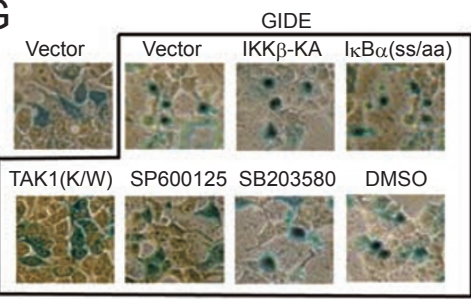

I

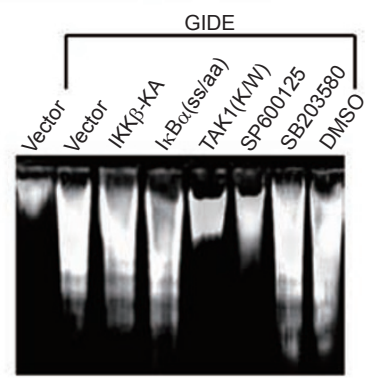

B
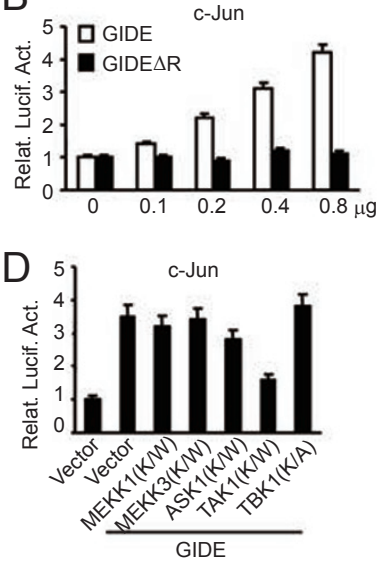

F

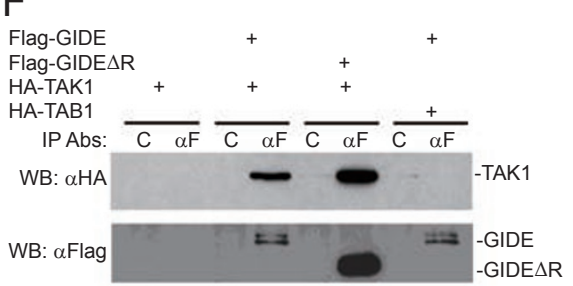

$\mathrm{H}$

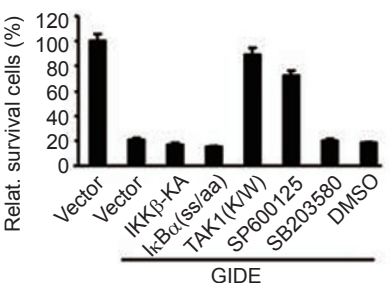

$J$

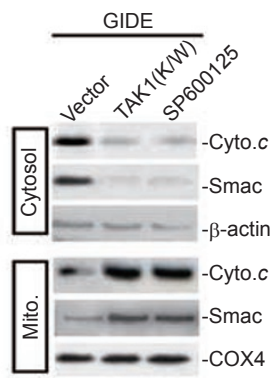

Figure 5 GIDE induces apoptosis through a JNK-dependent pathway. (A) Effects of GIDE and GIDE $\Delta R$ on NF-kB activation. 293 cells $\left(\sim 1 \times 10^{5}\right)$ were transfected with $0.1 \mu \mathrm{g}$ of NF-kB-luciferase reporter plasmid and the indicated amounts (in $\mu \mathrm{g}$ ) of expression plasmids. Reporter assays were performed $16 \mathrm{~h}$ after transfection. (B-C) Effects of GIDE and its mutants on JNK activation. 293 cells were transfected with GIDE and its mutants as indicated. C-Jun reporter assays (B) or in vitro kinase assays with GST-C-Jun as substrate (C) were performed as described [31]. (D) GIDE-induced JNK activation is inhibited by a TAK1 kinase-inactive mutant. 293 cells were transfected with GIDE and the indicated kinase-inactive mutants. C-Jun reporter assays were performed as described [47]. (E) GIDE does not activate CHOP or Elk-1. 293 cells were transfected with GIDE or MEKK3. CHOP and Elk-1 reporter assays were performed as described [31]. (F) GIDE interacts with TAK1. 293 cells were transfected with the indicated plasmids. Cell lysates were immunoprecipitated with anti-FLAG or control mouse IgG. The immunoprecipitates were analyzed by western blot with anti-HA (upper panel) and anti-Flag (lower panel) antibodies. (G-I) Effects of various mutants or small molecule inhibitors on GIDE-induced apoptosis. 293 cells were transfected with the indicated plasmids, treated with SP600125 (20 $\mu \mathrm{M})$, SB203580 $(20 \mu \mathrm{M})$, or left untreated, as indicated, for $16 \mathrm{~h}$. The cells were stained with X-gal $(\mathbf{G})$, the number of surviving blue cells was counted $(\mathbf{H})$, or DNA fragmentation experiments were performed (I). (J) JNK is essential for GIDE-induced cytochrome $c$ and Smac release. 293 cells were transfected with GIDE plus control vector or TAK1 $(\mathrm{K} / \mathrm{W})$, or treated with SP600125 $(20 \mu \mathrm{M})$ for $16 \mathrm{~h}$. The cells were fractionated and analyzed by western blots with the indicated antibodies. 


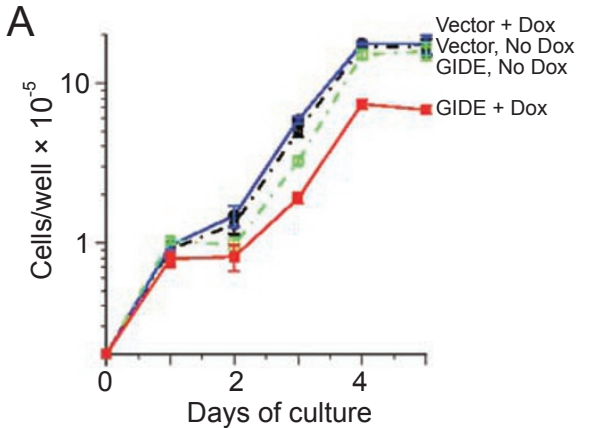

B
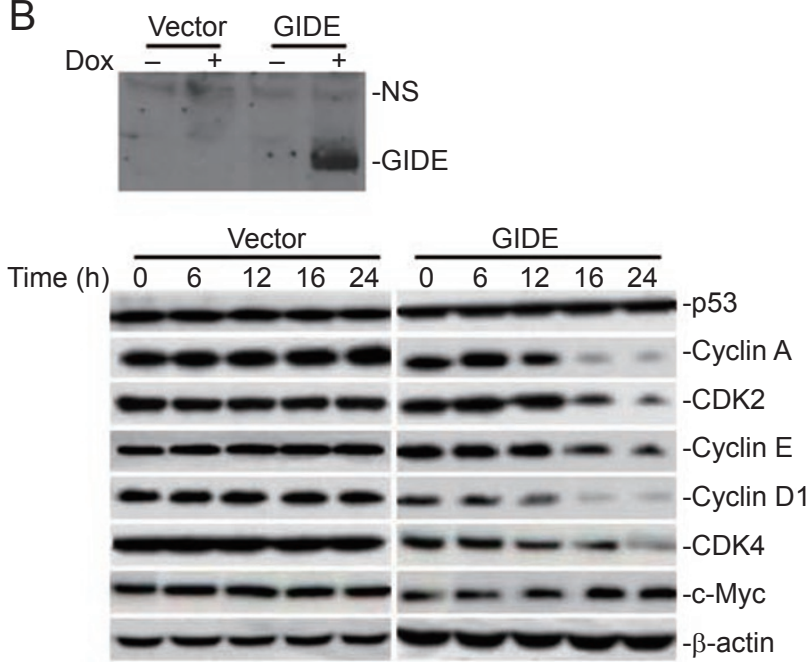

Figure 6 Inducible expression of GIDE inhibits cell growth. (A) NIH3T3 tet-on cells were transfected with empty vector (Vector) or GIDE (GIDE), together with pTK-Hyg. The cells were selected with hygromycin $(500 \mu \mathrm{g} / \mathrm{ml})$ for 15 days. Stable GIDE-expressing clones were induced with doxycycline or left untreated, and cell growth was monitored by counting every day for 5 days. Results shown are the means and standard errors. Similar results were obtained with an independent clone of 3T3 cells overexpressing GIDE. (B) Induction of GIDE expression in GIDE-transfected 3T3 cells was confirmed by western blot analysis with anti-GIDE antibody. (C) 293 cells were transfected with GIDE or control vector for the indicated times. Levels of the indicated cell cycle-related proteins were examined by western blots.

determined which event is required for GIDE-induced apoptosis. In apoptosis assays, overexpression of IKK $\beta$ $\mathrm{KA}$ and $\mathrm{I}_{\kappa} \mathrm{B} \alpha(\mathrm{SS} / \mathrm{AA})$, which inhibit GIDE-induced NF$\kappa \mathrm{B}$ (data not shown), did not inhibit GIDE-induced apoptosis and DNA fragmentation (Figure 5G). In contrast, overexpression of the TAK1 kinase-inactive mutant, TAK1(K/W), dramatically inhibited GIDE-induced apoptosis and DNA fragmentation (Figure 5G-5I). Moreover, the JNK-specific small molecular inhibitor SP600125, but not the p38 inhibitor SB203590 or carrier DMSO, also inhibited GIDE-induced apoptosis and DNA fragmentation (Figure 5G-5I). Consistently, TAK1(K/W) and
SP600125 blocked GIDE-induced release of cytochrome $c$ and Smac into the cytosol (Figure 5J). These data suggest that $\mathrm{JNK}$, but not NF- $\kappa \mathrm{B}$, activation is required for GIDE-induced release of cytochrome $c$ and Smac into the cytosol and subsequent apoptosis.

Over- or underexpression of GIDE inhibits or stimulates cell growth, respectively
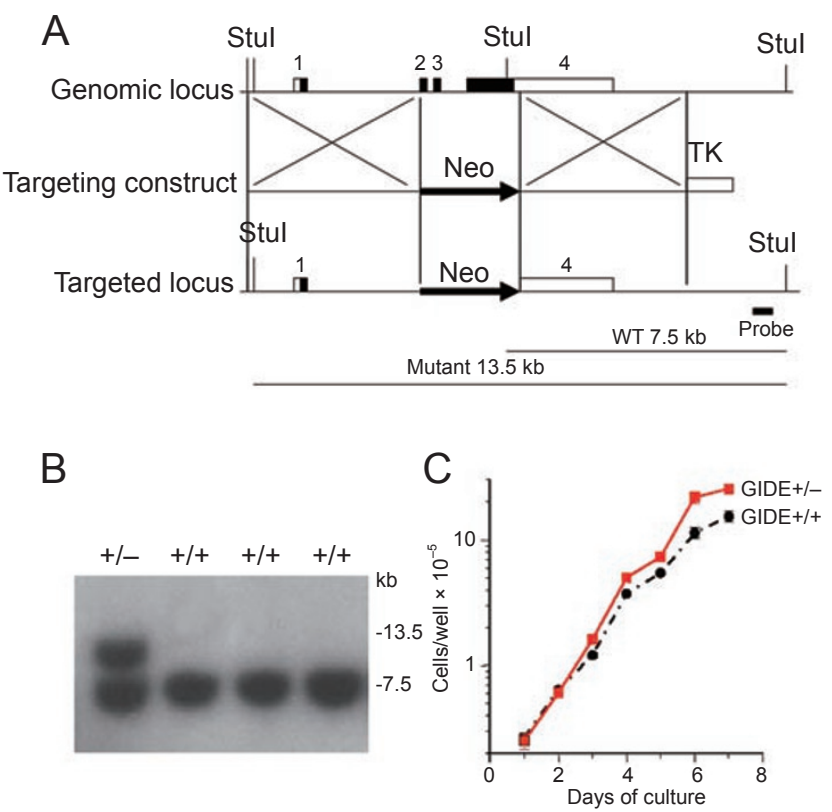

Figure 7 Under-expression of GIDE stimulates cell growth. (A) Targeting strategy for the GIDE locus. Boxes represent exons 1-4 of the mouse GIDE gene. Empty boxes represent the 5' untranslated region and filled boxes represent the coding region. Following homologous recombination, the exon 2, 3 and 4-containing coding region was replaced with the neomycin resistance gene $(\mathrm{NeO})$ on the targeted allele. Thymidine kinase from the herpes simplex virus (HSV-TK) was used for negative selection. GIDE was isolated from genomic DNA extracted from embryonic stem cells (TC1) by $\mathrm{PCR}$. The targeting vector was constructed by replacing a $2.1-\mathrm{kb}$ fragment encoding the GIDE open reading frame (exons 2, 3 and 4) with a Neo gene cassette, and HSV-TK was inserted into the genomic fragment for negative selection. The targeting construct was linearized by Sall restriction digestion and electroporated into TC1 ES cells (derived from 129Sv mouse strain). G418 and gancyclovir doubly-resistant colonies were selected and screened by Southern blot analysis. One of these colonies was found to contain the correctly targeted allele. (B) Genotyping of GIDE-deficient TC1 ES cell clones. Genomic DNA from TC1 ES cell clones was digested with Stul and hybridized to the probe. Results for wild type $(+/+)$ and heterozygous (+/-) GIDE-deficient TC1 ES cell clones are shown. (C) GIDE +/+ and +/- ES cells were plated on irradiated feeder cells. The cells, in triplicate cultures, were counted daily thereafter, with the feeder cells excluded based on their different cell size. Results shown are the means and standard errors of one of four experiments. 
As described above, GIDE potently induces apoptosis when overexpressed in 293 or other cells. To determine the effect of GIDE when expressed at relatively lower levels, we generated GIDE-inducible NIH3T3 cell lines. Inducible expression of GIDE in NIH3T3 cells caused apoptosis of only a small fraction (1-5\%) of cells, as suggested by annexin 5 staining (data not shown). Instead, ectopic induction of GIDE expression by doxcycline inhibited the growth of NIH3T3 cells, increasing their cycle time from about 15.4 to $19.2 \mathrm{~h}$ (Figure 6A and 6B). In accord with this result, overexpression of GIDE caused degradation of cyclin A, E and D1, as well as CDK2 and CDK4, but not p53 and c-Myc (Figure 6C). To find out if the opposite result applied in cells underexpressing GIDE, we measured the growth rates of embryonic stem (ES) cells heterozygous for a deletion of the GIDE gene (Figure 7A and 7B). The GIDE ${ }^{+/}$cells grew faster than $G I D E^{+/+}$cells, with cycle times of $18.6 \mathrm{~h}$, versus $21.8 \mathrm{~h}$ (Figure 7C). These data suggest that GIDE can inhibit cell growth, probably by promoting degradation of cyclins and CDKs.

\section{Discussion}

Here we report the identification of GIDE, an E3 ubiquitin ligase that, when overexpressed, induces apoptosis through novel caspase-9 and JNK activation-dependent pathways. When less overexpressed, GIDE slows cell growth rates and, when underexpressed, accelerates the cell cycle. This phenomenon may explain why, despite the fact that we have chimeric founders generated from $G I D E^{+/-}$ES cells, we have been unable to obtain heterozygous progeny from these mice (data not shown). The aberrant growth rate caused by heterozygous deletion of GIDE may be lethal at an early stage of embryonic development.

GIDE contains a C-terminal RING finger domain, which is mostly conserved with those of the IAP family members (Figure 1). The RING finger domain of GIDE is required for its E3 ligase activity and auto-ubiquitination (Figure 2). Aside from the RING finger domain, GIDE shows no conservation with known proteins, suggesting that GIDE may function through novel mechanisms. GIDE contains two transmembrane regions and localizes in the mitochondria. Overexpression of GIDE activates $\mathrm{JNK}$, an event that precedes release of cytochrome $c$ and Smac as well as apoptosis. These phenomena require GIDE's E3 ligase domain. In addition, caspase inhibitors crmA and z-VAD-fmk, as well as XIAP and an inactive mutant of caspase-9, block GIDE-induced apoptosis (Figure 4). These results suggest that GIDE induces caspase-dependent apoptotic pathways. Thus, our find- ings suggest that the E3 ubiquitin ligase GIDE induces apoptosis through activation of the protein kinase JNK and subsequent activation of caspases. The processes by which GIDE slows cell growth are currently unknown.

Other than itself, the physiological substrates for GIDE are unknown. We examined whether GIDE could ubiquitinate several candidate proteins, including XIAP; Survivin, an IAP family member that is highly expressed in cancer cells but not in normal cells and has an antiapoptotic role; cFLIP, an anti-apoptotic protein that is also a substrate of the E3 ligase Itch and TAK1, a kinaseactivating JNK. The results indicated that none of these proteins was a GIDE substrate (data not shown).

In co-immunoprecipitation experiments, GIDE interacted with the MAP3K family member TAK1 (Figure 5F). A kinase-inactive mutant of TAK1, but not mutants of MEKK1, MEKK3, ASK1 and TBK1, inhibited GIDEinduced JNK activation (Figure 5D). These results suggest that TAK1 is specifically involved in GIDE-induced JNK activation. GIDE-induced TAK1 and JNK activation requires GIDE's C-terminal RING finger domain, which confers its autoubiquitination activity (Figure 5). Although the exact mechanisms are unclear, GIDE-mediated TAK1 activation may be similar to the activation of TAK1 by TRAF6. TRAF6 is an E3 ubiquitin ligase that is critically involved in IL-1- and Toll-like receptor-induced NF- $\kappa$ B and JNK activation pathways [25]. In these pathways, TRAF6, which signals through TAK1 and the auto-ubiquitination of TRAF6, is sufficient to trigger the activation of TAK1 [25]. XIAP also interacts with TAK1, and may activate TAK1 through a similar mechanism.

JNK may promote apoptosis by engaging the mitochondrial-dependent intrinsic pathway. It has been shown that UV-induced Bax activation and cytochrome $c$ release are absent in JNK1/JNK2-deficient MEFs, and Bax and Bak double-deficient cells are resistant to apoptosis induced by UV and JNK activation [26-28]. It has also been shown that JNK-dependent phosphorylation of the $\mathrm{BH} 3$ proteins Bim and $\mathrm{Bmf}$ causes their dissociation from dynein and myosin motor complexes, and, subsequently, these dissociated proteins induce apoptosis through Bax and Bak [28]. Recently, it has been shown that JNK activation leads to phosphorylation of 14-3-3 proteins, which causes dissociation of 14-3-3 proteins with Bax. The released Bax is translocated to mitochondria, where it triggers the intrinsic apoptotic pathway [29]. Moreover, in RelA-deficient MEFs or human HeLa cells expressing an $\mathrm{I}_{\kappa} \mathrm{B} \alpha$ mutant that blocks NF- $\kappa \mathrm{B}$ activation, activation of JNK causes processing of Bid into tBid by an unknown mechanism. The processed tBid is translocated to the mitochondria, where it selectively triggers release of Smac, but not of cytochrome $c$. The 
released Smac causes activation of caspase- 8 and promotes apoptosis [13]. Based on these previous studies, it is possible that GIDE-induced apoptosis is also mediated by the 14-3-3 and Bcl2 family members. However, when overexpressed in Bax and Bak double knockout fibroblasts, GIDE could still induce apoptosis (data not shown), which is consistent with the observation that Bcl-XL and 14-3-3 could not block GIDE-induced apoptosis (Figure 3). In addition, although XIAP could block GIDE-induced apoptosis, GIDE could not ubiquitinate XIAP or cause XIAP degradation (data not shown), indicating that XIAP is not a direct target of GIDE. Thus, our findings suggest that GIDE induces a novel, non-classic apoptotic pathway.

Northern blot and western blot analysis suggests that GIDE is expressed in most normal human tissues at relatively high levels (Figure 2). However, we failed to detect GIDE expression in eight examined cancer cell lines, including five lung-derived (H549, H441, H290, H157, and 16HBE), one each of liver- (Hep3B), colon(H1299) and kidney- (293) derived cancer cell lines (data not shown). Together with our observations that GIDE induces apoptosis or inhibits cell growth when ectopically expressed in immortal cell lines, these observations suggest the possibility that GIDE is a tumor suppressor gene that is down-regulated in cancer cells.

\section{Materials and Methods}

\section{Reagents \\ Antibodies against Flag and HA epitopes (Sigma); ubiquitin, PARP, cyclin A, cyclin D1, cyclin E, CDK2,4 and $\beta$-actin (Santa Cruz Biotechnology); cytochrome $c$, Smac, caspase-7,8,9 and p53 $\beta$ (Cell Signaling Technology); Cox4 (Molecular Probes); z-VAD- fmk (R\&D systems); Rh-123, SP600125 and SB203580 (Sigma); and human embryonic kidney 293 cells (ATCC, Manassas, VA) were purchased from the indicated manufacturers. NIH3T3 Tet- on cell line was provided by Dr James Hagman (National Jewish Medical and Research Center).}

\section{Constructs}

Mammalian expression plasmids for $I_{\kappa} \mathrm{B} \alpha(\mathrm{SS} / \mathrm{AA})$, FADDDN, crmA, TAK1(K/W), TBK1(K/W), ASK1(K/W), MEKK1(K/ W), MEKK3, MEKK3(K/W), HA-TAK1, HA-TAB1, cIAP1, p53-DN, Bcl-XL, Caspase 8(c360s) and Caspase 9(c276s) were described previously [30-32]. Mammalian expression plasmids for HA- or FLAG-tagged GIDE, GIDE $\Delta$ R, GIDE(H319A), XIAP, 14-3-3 $\sigma$-DN and Caspase-9(c276s) were constructed by PCR amplification of the corresponding cDNA fragments and subsequently cloned into a CMV promoter-based vector containing an N-terminal HA or FLAG tag.

\section{Northern blot}

Human multiple tissue mRNA blots were purchased from Clontech (Palo Alto, CA). The blots were hybridized with
${ }^{32} \mathrm{P}$-labeled GIDE cDNA in Rapid Hybridization Buffer (Clontech, Palo Alto, CA) under high-stringency conditions.

Transfection, co-immunoprecipitation, reporter gene, immunofluorescence, in vitro kinase, apoptosis assays and production of heterozygous ES cells

The methods used were as described previously [30-32].

\section{DNA fragmentation assays}

Transfected cells were washed twice with cold PBS and lysed with $2 \% \mathrm{NP}-40$ containing $0.2 \mathrm{mg} / \mathrm{ml}$ Proteinase $\mathrm{K}$. DNA in the lysate was precipitated with 2 volumes of ethanol. The pellets were dissolved in $\mathrm{H}_{2} \mathrm{O}$ and analyzed by electrophoresis in a $2 \%$ gel.

\section{Mitochondrial membrane potential assays}

293 cells $\left(\sim 2 \times 10^{5}\right)$ were transfected with $1 \mu \mathrm{g}$ of expression plasmids for the indicated time. The transfected cells were harvested and washed with $\operatorname{PBS}(+)$ three times and stained with $\mathrm{Rh} 123(10 \mu \mathrm{g} / \mathrm{ml})$ at $37^{\circ} \mathrm{C}$ for $30 \mathrm{~min}$, then washed three times with PBS $(+)$. The mitochondrial membrane potential was analyzed by flow cytometry.

\section{Induced expression of GIDE in NIH3T3 tet-on cells}

NIH3T3 tet-on cells were transfected with pTRE-HA-GIDE (or pTRE) and pTK-Hyg in the ratio 10:1 by Lipofectamine 2000 following the manufacturer's instructions (Invitrogen, Carlsbad, CA). One day after transfection, cells were selected with hygromycin $(500 \mu \mathrm{g} / \mathrm{ml})$ for 15 days. Individual clones were induced with doxcycline $(1 \mu \mathrm{g} / \mathrm{ml})$, and induction of GIDE expression was confirmed by western blot.

\section{Growth curves}

The growth rate of stable clones of transfected NIH3T3 tet-on cells was measured by counting the number of cells. Cells were subcultured into six-well plates at a density of $1.5 \times 10^{4}$ cells/well in medium with or without doxcycline $(1 \mu \mathrm{g} / \mathrm{ml})$. The medium was replaced with fresh medium every 2 days. The cells were trypsinized and counted every $24 \mathrm{~h}$ for 5 days. Similar assays were performed for $G I D E^{+/+}$and $G I D E^{+/-}$ES cells, except that doxycyclin was not added, and the cells were grown on irradiated feeder cells.

\section{Acknowledgments}

The authors thank Saiphone Webb for her help in production and analysis of the $G I D E^{+/}$ES cells. This work was supported by Hi-Tech Research and Development Program of China (863 program, Grant No. 2006AA02A306), the Chinese 111 project (Grant No. B06018), and National Institutes of Health (Grant No. CA108771).

\section{References}

1 Earnshaw WC, Martins LM, Kaufmann SH. Mammalian caspases: structure, activation, substrates, and functions during apoptosis. Annu Rev Biochem 1999; 68:383-424.

2 Green DR, Reed JC. Mitochondria and apoptosis. Science 
1998; 281:1309-1312.

3 Kroemer G, Martin SJ. Caspase-independent cell death. Nat Med 2005; 11:725-730.

4 Salvesen GS, Dixit VM. Caspase activation: the induced-proximity model. Proc Natl Acad Sci USA 1999; 96:10964-10967.

5 Hill MM, Adrain C, Martin SJ. Portrait of a killer: the mitochondrial apoptosome emerges from the shadows. Mol Interv 2003; 3:19-26.

6 Du C, Fang M, Li Y, Li L, Wang X. Smac, a mitochondrial protein that promotes cytochrome c-dependent caspase activation by eliminating IAP inhibition. Cell 2000; 102:33-42.

7 Olson MR, Holley CL, Yoo SJ, Huh JR, Hay BA, Kornbluth $\mathrm{S}$. Reaper is regulated by IAP-mediated ubiquitination. $J$ Biol Chem 2003; 278:4028-4034.

8 Hegde R, Srinivasula SM, Datta P, et al. The polypeptide chain-releasing factor GSPT1/eRF3 is proteolytically processed into an IAP-binding protein. J Biol Chem 2003; 278:38699-38706.

9 Duckett CS. IAP proteins: sticking it to Smac. Biochem J 2005; 385:e1-e2.

10 Deveraux QL, Takahashi R, Salvesen GS, Reed JC. X-linked IAP is a direct inhibitor of cell-death proteases. Nature 1997; 388:300-304.

11 Suzuki Y, Nakabayashi Y, Takahashi R. Ubiquitin-protein ligase activity of $\mathrm{X}$-linked inhibitor of apoptosis protein promotes proteasomal degradation of caspase- 3 and enhances its anti-apoptotic effect in Fas-induced cell death. Proc Natl Acad Sci USA 2001; 98:8662-8667.

12 Wilkinson JC, Wilkinson AS, Scott FL, Csomos RA, Salvesen GS, Duckett CS. Neutralization of Smac/Diablo by inhibitors of apoptosis (IAPs). A caspase-independent mechanism for apoptotic inhibition. J Biol Chem 2004; 279:51082-51090.

13 Deng Y, Ren X, Yang L, Lin Y, Wu X. A JNK-dependent pathway is required for TNFalpha-induced apoptosis. Cell 2003; 115:61-70.

14 Johnson GL, Lapadat R. Mitogen-activated protein kinase pathways mediated by ERK, JNK, and p38 protein kinases. Science 2002; 298:1911-1912.

15 Kockel L, Homsy JG, Bohmann D. Drosophila AP-1: lessons from an invertebrate. Oncogene 2001; 20:2347-2364.

16 Salvesen GS, Duckett CS. IAP proteins: blocking the road to death's door. Nat Rev Mol Cell Biol 2002; 3:401-410.

17 Xia Z, Dickens M, Raingeaud J, Davis RJ, Greenberg ME. Opposing effects of ERK and JNK-p38 MAP kinases on apoptosis. Science 1995; 270:1326-1331.

18 Hochedlinger K, Wagner EF, Sabapathy K. Differential effects of JNK1 and JNK2 on signal specific induction of apoptosis. Oncogene 2002; 21:2441-2445.

19 Sabapathy K, Kallunki T, David JP, Graef I, Karin M, Wagner EF. c-Jun NH2-terminal kinase (JNK)1 and JNK2 have similar and stage-dependent roles in regulating $\mathrm{T}$ cell apoptosis and proliferation. J Exp Med 2001; 193:317-328.

20 Sabapathy K, Jochum W, Hochedlinger K, Chang L, Karin M, Wagner EF. Defective neural tube morphogenesis and altered apoptosis in the absence of both JNK1 and JNK2. Mech Dev 1999; 89:115-124.

21 Matsuda A, Suzuki Y, Honda G, et al. Large-scale identification and characterization of human genes that activate NFkappaB and MAPK signaling pathways. Oncogene 2003; 22:3307-3318.

22 Hofer-Warbinek R, Schmid JA, Stehlik C, Binder BR, Lipp J, de Martin R. Activation of NF-kappa B by XIAP, the X chromosome-linked inhibitor of apoptosis, in endothelial cells involves TAK1. J Biol Chem 2000; 275:22064-22068.

23 Sanna MG, da Silva Correia J, Ducrey O, et al. IAP suppression of apoptosis involves distinct mechanisms: the TAK1/ JNK1 signaling cascade and caspase inhibition. Mol Cell Biol 2002; 22:1754-1766.

24 Baud V, Liu ZG, Bennett B, Suzuki N, Xia Y, Karin M. Signaling by proinflammatory cytokines: oligomerization of TRAF2 and TRAF6 is sufficient for JNK and IKK activation and target gene induction via an amino-terminal effector domain. Genes Dev 1999; 13:1297-1308.

25 Wang C, Deng L, Hong M, Akkaraju GR, Inoue J, Chen ZJ. TAK 1 is a ubiquitin-dependent kinase of MKK and IKK. $\mathrm{Na}$ ture 2001 ; 412:346-351.

26 Lei K, Nimnual A, Zong WX, et al. The Bax subfamily of $\mathrm{Bcl} 2$-related proteins is essential for apoptotic signal transduction by c-Jun NH(2)-terminal kinase. Mol Cell Biol 2002; 22:4929-4942.

27 Wei MC, Zong WX, Cheng EH, et al. Proapoptotic BAX and BAK: a requisite gateway to mitochondrial dysfunction and death. Science 2001; 292:727-730.

28 Lei K, Davis RJ. JNK phosphorylation of Bim-related members of the Bcl2 family induces Bax-dependent apoptosis. Proc Natl Acad Sci USA 2003; 100:2432-2437.

29 Tsuruta F, Sunayama J, Mori Y, et al. JNK promotes Bax translocation to mitochondria through phosphorylation of 14-3-3 proteins. EMBO J 2004; 23:1889-1899.

$30 \mathrm{Hu}$ WH, Johnson H, Shu HB. Tumor necrosis factor-related apoptosis-inducing ligand receptors signal NF-kappaB and JNK activation and apoptosis through distinct pathways. $J$ Biol Chem 1999; 274:30603-30610.

31 Xu LG, Li LY, Shu HB. TRAF7 potentiates MEKK3-induced AP1 and CHOP activation and induces apoptosis. $\mathrm{J} \mathrm{Biol} \mathrm{Chem}$ 2004; 279:17278-17282.

32 Huang J, Liu T, Xu LG, Chen D, Zhai Z, Shu HB. SIKE is an IKK epsilon/TBK1-associated suppressor of TLR3- and virus-triggered IRF-3 activation pathways. EMBO J 2005; 24:4018-4028. 